\title{
Nonequilibrium Attractor in High-Temperature QCD Plasmas
}

\author{
Dekrayat Almaalol, ${ }^{1}$ Aleksi Kurkela, ${ }^{2,3}$ and Michael Strickland $\oplus^{1}$ \\ ${ }^{1}$ Department of Physics, Kent State University, Kent, Ohio 44242, USA \\ ${ }^{2}$ Theoretical Physics Department, CERN, 1211 Genéve 23, Switzerland \\ ${ }^{3}$ Faculty of Science and Technology, University of Stavanger, 4036 Stavanger, Norway
}

(Received 20 April 2020; revised 17 August 2020; accepted 20 August 2020; published 17 September 2020)

\begin{abstract}
We establish the existence of a far-from-equilibrium attractor in weakly coupled gauge theory undergoing one-dimensional Bjorken expansion. We demonstrate that the resulting far-from-equilibrium evolution is insensitive to certain features of the initial condition, including both the initial momentumspace anisotropy and initial occupancy. We find that this insensitivity extends beyond the energymomentum tensor to the detailed form of the one-particle distribution function. Based on our results, we assess different procedures for reconstructing the full one-particle distribution function from the energy-momentum tensor along the attractor and discuss implications for the freeze-out procedure used in the phenomenological analysis of ultrarelativistic nuclear collisions.
\end{abstract}

DOI: 10.1103/PhysRevLett.125.122302

Fluid-dynamic description is a powerful tool in the phenomenological analysis of ultrarelativistic nuclear collisions [1-3]. In a fluid-dynamic description of the evolution of the collision system, only a small subset of the degrees of freedom are dynamically evolved. These are quantities derived from the energy-momentum tensor $T^{\mu \nu}$, namely, local temperatures, velocities and, in the case of viscous hydrodynamics, information about the shear and bulk viscous tensors. The experiments do not, however, measure fluid-dynamic variables, but rather distributions of particles that have "frozen out" and free stream to the detectors - the angular and momentum distributions of these particles inform us about the material properties of the fluid created [4]. To convert fluid-dynamic fields to particle distributions, a freeze-out procedure has to be applied. While the energy-momentum tensor depends only on the first momentum-integral moments of the distribution function, the particle distributions contain information about all the moments. Therefore, the conversion of the fluid-dynamic information to particle distributions necessarily involves injection of new information in the form of theoretical assumptions. The common procedure is to assume that the distribution function has a nearequilibrium form whose deviations from equilibrium arise from formally small corrections-the shape of the corrections is determined by the response of a linearized collision

Published by the American Physical Society under the terms of the Creative Commons Attribution 4.0 International license. Further distribution of this work must maintain attribution to the author(s) and the published article's title, journal citation, and DOI. Funded by SCOAP ${ }^{3}$. kernel in some assumed kinetic theory to an infinitesimal strain $[5,6]$.

As the freeze-out procedure strongly affects the phenomenological analysis and conclusions about the matter created in ultrarelativistic heavy-ion collisions, it is of great interest to scrutinize quantitatively how well justified are the theoretical assumptions about the shape of the nonequilibrium distribution functions. The need for such scrutiny becomes increasingly important in the case of small systems, e.g., peripheral nucleus-nucleus collisions, proton-nucleus, and high-multiplicity protonproton collisions where fluid-dynamical description is being applied to situations which most likely remain far from equilibrium throughout their dynamical evolution.

There has been a large body of work quantifying to what extent various different formulations of viscous fluid dynamics are able to reproduce the time evolution of the components of the energy-momentum tensor undergoing expansion in various geometries. In particular, it has been observed that the hydrodynamic constitutive equations that relate the stress tensor to gradients of the flow fields are well satisfied in systems which are still far from equilibrium at least in systems characterized by flow with a large degree of symmetry $[7,8]$ (for systems with less symmetry cf. Ref. [9-12]) - a feat dubbed hydrodynamization without thermalization [13-35]. It has also been observed that many microscopic models, as well as various formulations of fluid dynamics, exhibit rapid information loss of some details (in particular the initial longitudinal pressure) of the initial condition leading to nonequilibrium attractor behavior. The qualitative similarity of the attractors between different theories has been advocated to extend the applicability of the fluiddynamic models to far-from-equilibrium regimes where the 
ordinary justification of fluid dynamics as a nearequilibrium expansion is questionable.

Much less attention has been paid to the validity of the freeze-out procedure far from equilibrium. One of the challenges is that models with trivial momentum dependence, such as kinetic theory in relaxation time approximation, can give only limited information about the validity of the freeze-out procedure, whereas strongly coupled models without quasiparticle structure do not even possess underlying particle distributions. Here, we discuss the reconstruction of the particle distributions from the energy-momentum tensor in the effective kinetic theory (EKT) for weak-coupling quantum chromodynamics (QCD) that becomes leading-order accurate in the limit of high center-of-mass energy collisions [36]. The rich momentum-dependent structure of the EKT collision kernel allows for a nontrivial test of the freeze-out procedure in this theoretically clean limit. We follow $0+1 d$ far-from-equilibrium Bjorken flow within this model and compare different moments of the distribution function to those predicted by hydrodynamic freeze-out prescriptions. We find that EKT seems to exhibit qualitatively similar far-from-equilibrium attractor behavior to kinetic theory in the relaxation time approximation (RTA) and Israel-Stewart-type hydrodynamics $[13,17,32]$ both at early (early-time or pullback attractor) and late times (latetime or hydrodynamic attractor). We find that this attractive behavior is not restricted to the components of the energy-momentum tensor but extends to other integral moments as well. We observe that the commonly used freeze-out prescriptions reproduce low-order moments of the distribution well at late times, however, they can fail at early times or when considering moments sensitive to momenta much larger than the temperature. We discuss the implications for phenomenological fluid-dynamic modeling of small collision systems such as $p p$ and $p A$.

Methodology.-We make use of a numerical implementation of the effective kinetic theory of Refs. [8,36,37]. In parametrically isotropic systems, EKT gives a leading order accurate description (in $\alpha_{s}$ ) of the QCD time evolution of the one-particle distribution function and allows for a numerical realization of the so-called bottom-up thermalization scenario [38]. In practice, we solve the EKT Boltzmann equation for a gluonic plasma undergoing one-dimensional Bjorken expansion with transverse translational symmetry such that the effective Boltzmann equation reads [39]

$$
-\frac{d f(\mathbf{p})}{d \tau}+\frac{p_{z}}{\tau} \partial_{p_{z}} f=\mathcal{C}_{1 \leftrightarrow 2}[f(\mathbf{p})]+\mathcal{C}_{2 \leftrightarrow 2}[f(\mathbf{p})],
$$

where $f(\mathbf{p})$ is the gluonic one-particle distribution function (per degree of freedom). The elastic scattering term $\mathcal{C}_{2 \leftrightarrow 2}$ and the effective inelastic term $\mathcal{C}_{1 \leftrightarrow 2}$ include physics of dynamical screening and Landau-Pomeranchuck-Migdal suppression [8,36,37].

For the numerical solution of Eq. (1), we discretize $n(\mathbf{p})=p^{2} f(\mathbf{p})$ on an optimized momentum-space grid and use Monte Carlo sampling to compute the integrals appearing in the elastic and inelastic collisional kernels. The algorithm used is based on Refs. [37,40] and exactly conserves energy while also exactly accounts for the particle number violation originating from the inelastic contributions to the collisional kernel. Because of the azimuthal symmetry of Bjorken flow, one can discretize momentum space on an effectively two-dimensional gridhere we use $250 \times 2000$ points in the $p$ and $x=\cos \theta$ directions, respectively. The momenta $p$ are distributed on a logarithmic grid in the ranges $[0.02,45] \Lambda$, where $\Lambda$ is the typical energy scale of the initial condition. In all figures presented herein, we used 't Hooft coupling $\lambda=N_{c} g^{2}=10$ corresponding to a specific shear viscosity of $\bar{\eta}=\eta / s \approx 0.62$ [14,41].

We follow the time evolution of a complete set of integral moments characterizing the momentum dependence of the distribution function [27]

$$
\mathcal{M}^{n m}(\tau) \equiv \int \frac{d^{3} p}{(2 \pi)^{3}} p^{n-1} p_{z}^{2 m} f(\tau, \mathbf{p}),
$$

where $p=|\mathbf{p}|$. Note that the energy density is given by $\varepsilon=\nu \mathcal{M}^{20}$, longitudinal pressure by $P_{L}=\nu \mathcal{M}^{01}$, and number density by $n=\nu \mathcal{M}^{10}$ for $\nu$ degrees of freedom ( $\nu=2 d_{A}$ for $d_{A}$ adjoint colors of gluons). The other moments do not have an interpretation in terms of the usual hydrodynamic moments considered in the literature, although the $m=0$ modes are simply related to the effective temperatures introduced in Refs. [42,43].

In our results, these moments will be scaled by their corresponding equilibrium values with $\overline{\mathcal{M}}^{n m}(\tau) \equiv$ $\mathcal{M}^{n m}(\tau) / \mathcal{M}_{\mathrm{eq}}^{n m}(\tau)$, where, using a Bose distribution, one obtains

$$
\mathcal{M}_{\mathrm{eq}}^{n m}=\frac{T^{n+2 m+2} \Gamma(n+2 m+2) \zeta(n+2 m+2)}{2 \pi^{2}(2 m+1)} .
$$

The temperature $T$ here corresponds to the temperature of an equilibrium system with the same energy density, given by $T=\left(30 \varepsilon / \nu \pi^{2}\right)^{1 / 4}$.

The different moments are sensitive to different momentum regions of the distribution function and for future comparisons, we note that, in equilibrium, the typical momentum contributing to a given moment is $\langle p\rangle_{\mathrm{eq}}^{n m}=\mathcal{M}_{\mathrm{eq}}^{n+1, m} / \mathcal{M}_{\mathrm{eq}}^{n m}$, giving, e.g., $\langle p\rangle_{\mathrm{eq}}^{10} \simeq 2.7 T$, $\langle p\rangle_{\mathrm{eq}}^{01}=\langle p\rangle_{\mathrm{eq}}^{20} \simeq 3.83 T,\langle p\rangle_{\mathrm{eq}}^{21} \simeq 5.95 T$, and $\langle p\rangle_{\mathrm{eq}}^{33} \simeq 11 T$.

Results.-In Figs. 1 and 2, we present results for the evolution of three scaled moments, $\overline{\mathcal{M}}^{01}, \overline{\mathcal{M}}^{21}$, and $\overline{\mathcal{M}}^{33}$, in panels (a), (b), and (c), respectively. These simulations have been initialized with either of the two following initial 

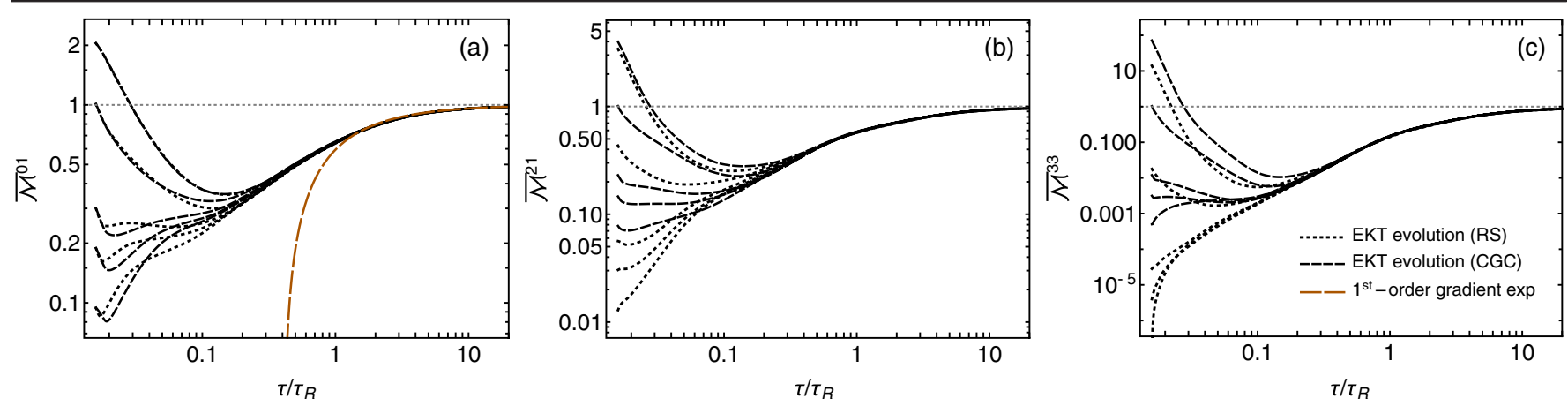

FIG. 1. Evolution of the scaled moments (a) $P_{L} / P_{L}^{\mathrm{eq}}=\overline{\mathcal{M}}^{01}$, (b) $\overline{\mathcal{M}}^{21}$, and (c) $\overline{\mathcal{M}}^{33}$ when varying the initial momentum-space anisotropy. Black dotted and dashed lines show EKT evolution with RS and CGC initial conditions, respectively. The orange longdashed line shows the first-order gradient expansion result (Navier-Stokes). See Supplemental Material, Fig. S1 [49] for plots of more moments.

conditions: (i) spheroidally deformed thermal initial conditions which we will refer to as "RS" initial conditions [44] and (ii) nonthermal color-glass-condenssate (CGC) inspired initial conditions [8]. In the first case, the initial gluonic one-particle distribution function is taken to be of the form

$$
f_{0, \mathrm{RS}}(\mathbf{p})=f_{\text {Bose }}\left(\sqrt{\mathbf{p}^{2}+\xi_{0} p_{z}^{2}} / \Lambda_{0}\right)
$$

where $-1<\xi_{0}<\infty$ encodes the initial momentum anisotropy and $\Lambda_{0}$ is a temperaturelike scale that sets the magnitude of the initial average transverse momentum. In the second case, we take for the form of the initial gluonic one-particle distribution

$$
f_{0, \mathrm{CGC}}(\mathbf{p})=\frac{2 A}{\lambda} \frac{\tilde{\Lambda}_{0}}{\sqrt{\mathbf{p}^{2}+\xi_{0} p_{z}^{2}}} e^{-\frac{2}{3}\left(\mathbf{p}^{2}+\xi_{0} \hat{p}_{z}^{2}\right) / \tilde{\Lambda}_{0}^{2}} .
$$

This form has been used in several earlier works (see, e.g., Refs. $[8,10,42,43,45])$, and is motivated by the saturation framework, where the initial average transverse momentum scale $\tilde{\Lambda}_{0}$ is related to the saturation scale $\tilde{\Lambda}_{0}=\left\langle p_{T}\right\rangle_{0} \approx$ $1.8 Q_{s}$ [46-48]. For LHC heavy-ion collisions one has $Q_{s} \sim \Lambda_{0} \sim 1-2 \mathrm{GeV}$. The overall constant $A$ is set by fixing the initial energy density to match an expectation $\tau_{0} \epsilon_{0}=$ $0.358 \nu Q_{s}^{3} / \lambda$ from a classical Yang-Mills simulation of Lappi [48].

In both figures, the dotted and dashed black lines correspond to EKT evolution using RS- and CGC-type initial conditions, respectively. Figure 1(a) shows the time evolution of the longitudinal pressure normalized by its equilibrium value. The different lines correspond to different initial $P_{L} / P_{L}^{\mathrm{eq}}=\overline{\mathcal{M}}^{01}$ with momentum-space anisotropy parameters $\xi_{0} \in\{-0.84,0,5.25,10.1,24\}$ at initial time $\tau_{0}=0.095(\nu / \varepsilon)^{1 / 4}$.

The integral moments are plotted as a function of a rescaled time variable $\tau / \tau_{R}(\tau)$, which measures the age of the system in units of the instantaneous interaction time
$\tau_{R}(\tau)$. As the density of the system changes, so does the interaction timescale, which is given by $\tau_{R}(\tau)=4 \pi \bar{\eta} / T(\tau)$. Scaling time in this manner guarantees that, as long as the system is described by hydrodynamics close to thermal equilibrium, $\overline{\mathcal{M}}^{01}$ will eventually be described by the firstorder gradient expansion, $\overline{\mathcal{M}}^{01}=1-\left(120 \zeta(5) / \pi^{5}\right) \tau_{R} / \tau$, at late times $[15,27]$. This is independent of the microscopic details or specific values of macroscopic hydrodynamic parameters. This fluid-dynamic behavior is seen in Fig. 1(a) where the evolutions of all the various different initial conditions eventually converge onto a universal curve- the late-time attractor (see also Refs. [51,52]). However, as observed also in other models [13,17,18,27,30,32,53], this collapse takes place before the system is well described by the hydrodynamic gradient expansion, the first order of which is shown in Fig. 1(a) as an orange dashed line.

While the late-time attractor behavior for the longitudinal pressure has been observed earlier in simplified kinetic theories, the solutions at hand allow us to study to what extent the attractive behavior determines the full overall shape of the distribution function. Our first main finding is shown in panels (b) and (c) of Fig. 1, which display the time evolution of two higher moments of the distribution function, $\overline{\mathcal{M}}^{21}$ and $\overline{\mathcal{M}}^{33}$. We observe that the higher moments collapse to a universal curve on the same timescale as $\overline{\mathcal{M}}^{01}$, demonstrating that the universality extends beyond simple hydrodynamic moments and it is the entire distribution as a function of $\mathbf{p}$ that reaches an attractor form. For corresponding results for a large set of moments see the Supplemental Material [49].

The timescale at which the different solutions to Eq. (1) collapse in Fig. 1 is roughly $\tau / \tau_{R} \sim 0.5$. While all theories must eventually collapse on a single curve, the time at which individual solutions collapse to the attractor depends on the details of the model. In Ref. [32] two qualitatively different patterns were identified. In kinetic theory with an RTA collisional kernel and second-order viscous hydrodynamics, the decay to the attractor took place by a power law whose scale was set by the initial time $\tau_{0}$ such that a 

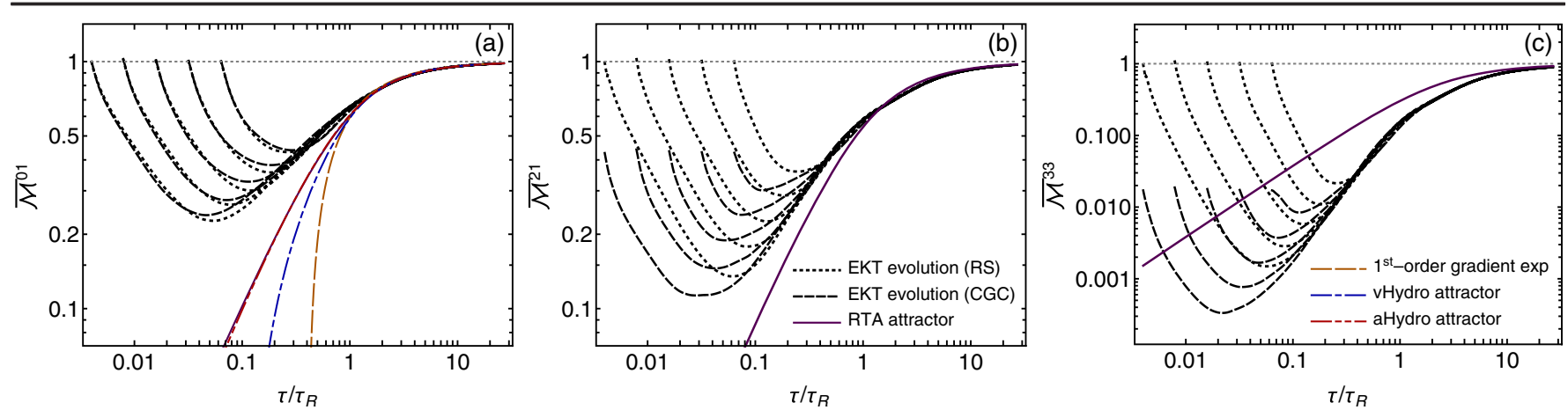

FIG. 2. Evolution of the scaled moments (a) $\overline{\mathcal{M}}^{01}$, (b) $\overline{\mathcal{M}}^{21}$, and (c) $\overline{\mathcal{M}}^{33}$ when varying the initialization time. Black dotted and dashed lines show EKT evolution with RS and CGC initial conditions, respectively. The purple solid line is the exact RTA attractor, the orange long-dashed line is the first-order gradient expansion result, the blue dot-dashed line is the DNMR vHydro attractor, and the red dot-dotdashed line is the aHydro attractor. See supplemental Fig. 1 for plots of more moments [49].

unique attractor exists at arbitrarily early time and can be found by studying initialization with decreasing $\tau_{0}$. In contrast, in AdS/CFT, a unique attractor exists only after a finite timescale (related to the decay of the so-called quasinormal modes $[13,32])$.

Figure 2 shows a set of solutions with fixed initial conditions (RS or CGC) with successively decreasing $\tau_{0}$. The figure demonstrates that earlier initializations lead to faster decay to the attractor signifying $\tau_{0}$ scaling of the decay and the existence of an early-time (or pullback [29]) attractor in EKT. We note that at late times the attractor for both overoccupied (CGC) and the thermal (RS) initial conditions are the same. This implies that upon reaching the attractor, the late-time evolution is not only insensitive to the initial longitudinal pressure of the initial condition but also to the initial occupancy and momentum profile for these physically motivated initial condition types and moderately large couplings $(\lambda=10)$. We note that in each run shown in Fig. 2 we observe a transition from purely free-streaming behavior to a collisionally broadened longitudinal expansion. This transition is related to the early stages of the bottom-up thermalization scenario [38,51], in particular, nonthermal attractors and associated prescaling behavior observed in prior EKT and classical Yang-Mills studies [54-58].

In Fig. 2(a) we also compare the EKT attractor to other known attractors for $P_{L} / P_{L}^{\mathrm{eq}}$. The solid purple line corresponds to the exact solution for the attractor in kinetic theory in the RTA approximation [27,32,59-62], the orange long-dashed line is the first-order gradient expansion result, the blue dot-dashed line corresponds to a formulation of viscous fluid dynamics used extensively in phenomenological description of heavy-ion collisions, namely, second-order viscous hydrodynamics (vHydro) of Denicol et al. [63,64], and the red dot-dot-dashed line corresponds to the anisotropic hydrodynamics (aHydro) attractor [23,65-68]. For details concerning how the attractors were determined in each case we refer the reader to the Supplemental Material [49]. We observe that while all of the attractors share some qualitatively similar features, the attractors of the different theories agree quantitatively only at $\tau / \tau_{R} \gg 1$ after the attractors follow the hydrodynamic gradient expansion. In particular, we emphasize that in vHydro the longitudinal pressure becomes negative at early times unlike in aHydro or EKT.

Panels (b) and (c) of Fig. 2 compare two higher-order moments of the RTA and EKT attractors. While the agreement between the two kinetic theories is rather good at late times for $n=0$ moments, the agreement becomes rapidly worse for increasing $n$ [49]. This implies that while the $|\mathbf{p}|$ dependence of the collision kernel may be rather well approximated by the simple RTA kernel at these values of coupling $\lambda$, the simplified angular structure of the RTA does not fully capture the shape of the longitudinal structure of the distribution function.

While the fluid-dynamic theories do not specify the higher moments of the distribution functions displayed in panels (b) and (c) of Fig. 2, it is a common practice to infer the full shape of the distribution from the shear components of the energy-momentum tensor only. For a given $T^{\mu \nu}$ the linearized viscous correction to the one-particle distribution function, $\delta f$ can be locally computed given an assumption of the collision kernel. Herein, we consider two possible forms for $\delta f$. The (i) quadratic Ansatz.

$$
\frac{\delta f_{(i)}}{f_{\mathrm{eq}}\left(1+f_{\mathrm{eq}}\right)}=\frac{3 \bar{\Pi}}{16 T^{2}}\left(p^{2}-3 p_{z}^{2}\right),
$$

which results from a wide set of models including RTA with momentum-independent relaxation time, momentum diffusion approximation, scalar field theory, and from EKT in the leading-log approximation [6]. Here $\bar{\Pi}=\Pi / \epsilon=1 / 3-$ $T^{z z} / \epsilon$ is the shear viscous correction to the longitudinal pressure scaled by the energy density. At full leading order, the EKT, however, has more structure; for large $p \gg T$, the EKT reduces to power law form of the (ii) LPM Ansatz. 

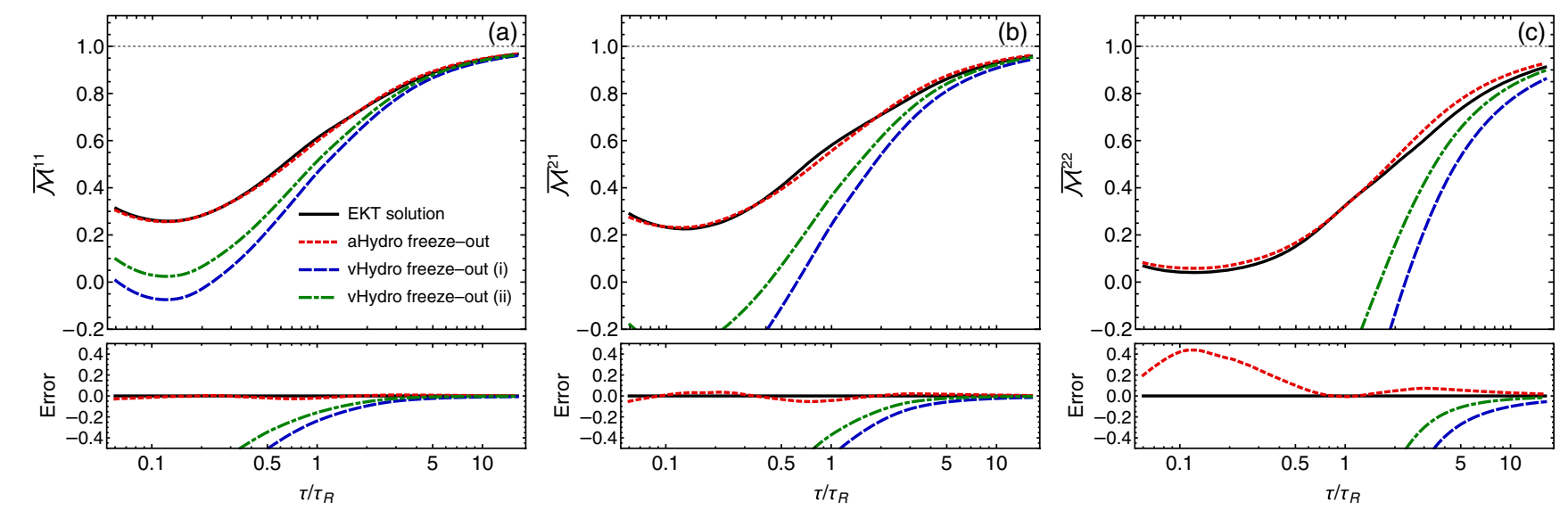

FIG. 3. Evolution of the scaled moments (a) $\overline{\mathcal{M}}^{11}$, (b) $\overline{\mathcal{M}}^{21}$, and (c) $\overline{\mathcal{M}}^{22}$. The black solid line is a typical EKT evolution, the reddashed line is the $P_{L}$-matched aHydro result for a given moment, the blue and green dot-dashed lines are the corresponding vHydro results using Eqs. (6) and (7), respectively. The relative error shown in the bottom panels is (approximation/EKT - 1). See Supplemental Material, Fig. S2 [49] for plots of more moments and comparison with our full set of runs.

$$
\frac{\delta f_{(i i)}}{f_{\mathrm{eq}}\left(1+f_{\mathrm{eq}}\right)}=\frac{16 \bar{\Pi}}{21 \sqrt{\pi} T^{3 / 2}}\left(p^{3 / 2}-\frac{3 p_{z}^{2}}{\sqrt{p}}\right) .
$$

This $p^{1.5}$ power law is numerically close to $\propto p^{1.38}$, which was found to describe the high-momentum region of the full EKT result [6].

Additionally, we consider a simple (iii) aHydro freezeout Ansatz procedure that does not assume linearization around equilibrium. Instead, in the aHydro freeze-out Ansatz, one assumes that the nonequilibrium distribution function can be approximated by a spheroidally deformed Bose distribution $f(p)=f_{\text {Bose }}\left(\sqrt{\mathbf{p}^{2}+\xi p_{z}^{2}} / \Lambda\right)[44,65,66]$. To test this approach, we fix $\xi$ locally such that the energymomentum tensor of the Ansatz matches with that of the full simulation and then make predictions for higher-order moments.

The different moments obtained by the above prescriptions are compared to the EKT attractor solution in Fig. 3 (see the Supplemental Material [49] for additional moments compared over our entire set of runs). At late times $\tau>5 \tau_{R}$, the low-order moments are described within a few percent by all the prescriptions, while some discrepancy remains even at $\tau \sim 20 \tau_{R}$ between the quadratic Ansatz (i) and our EKT results. The agreement worsens gradually at earlier times and around $\tau \sim \tau_{R}$ where the corrections to longitudinal pressure start to be sizable $P_{L} / P_{L}^{\mathrm{eq}} \sim 65 \%$, $\mathcal{M}^{11}$ exhibits an approximately $20 \%$ disagreement between EKT and both linearized Ansätze. The disagreement increases for higher moments and for earlier times. In contrast, we observe rather good agreement between the aHydro Ansatz and our EKT results at all times.

Conclusions and discussions.-An important step in the phenomenological analysis of nuclear collisions is the freeze-out procedure in which the hydrodynamical fields are converted to particle distributions. In the current phenomenological practice, the quadratic Ansatz (i) is widely used. This assumes linear deviations from thermal equilibrium, which is in stark contrast to the farfrom-equilibrium conditions in which fluid-dynamical modeling is practiced in current phenomenological applications, in particular in modeling of small systems (see, e.g., Refs. [69-74]). To address whether these linearized procedures remain quantitatively predictive far from equilibrium, we have confronted them with farfrom-equilibrium simulations of QCD effective kinetic theory. Our results in Fig. 3 show that at least in this simplified framework - admittedly quite far from the realities of phenomenological modeling-the nonlinear aHydro freeze-out Ansatz performed better in reconstructing moments of the distribution function compared to linearized Ansätze in far-from-equilibrium systems.

To translate the rescaled time variable $\tau / \tau_{R}$ to physical units in real-world LHC nuclear collisions at $\sqrt{s_{N N}}=5.02 \mathrm{TeV}$, we set the scale of the simulations by using the fact that the initial entropy density with $\nu_{\text {eff }} \simeq 40$ degrees of freedom satisfies $\tau_{0}^{\text {hydro }} s\left(\tau_{0}^{\text {hydro }}\right) \simeq$ $7.62 \mathrm{GeV}^{2}$, consistent with phenomenologically constrained values at LHC energies. For an initialization time of $\tau_{0}^{\text {hydro }}=0.25 \mathrm{fm} / \mathrm{c}$, this corresponds to an initial temperature of $T_{0}^{\text {hydro }} \approx 630 \mathrm{MeV}$ [68]. We also implicitly assume that the results as functions of $\tau / \tau_{R}$ do not depend on $\bar{\eta}$ as seen for the hydrodynamic moments in Ref. [10] and assume a value of $\bar{\eta} \approx 0.2$, which is consistent with the phenomenological extraction of the quantity.

Using this setup and averaging over our full set of runs, the rescaled times $\tau / \tau_{R}=\{0.2,0.5,1,2,5,10\}$ map to $\tau \simeq\{0.32,0.86,1.88,4.23,14.1,38.5\} \mathrm{fm} / \mathrm{c}$. This suggests that for $\tau_{\mathrm{fo}} \gg 5 \mathrm{fm} / \mathrm{c}$ the lowest-order modes, which are sensitive to $p \sim$ few $T$, can be well described by both aHydro and linearized freeze-out prescriptions. This implies that for central ultrarelativistic heavy-ion collisions one can have a faithful reproduction of the low-momentum 
part of the freeze-out distribution function. However, when considering higher moments, which are sensitive to higher momenta $\langle p\rangle^{n m}$, or applying early-time freeze-out for smaller systems such as peripheral nucleus-nucleus collisions and proton-nucleus collision, the aHydro freezeout Ansatz is favored.

In closing, we note that the current study was performed in a very simple setting with one-dimensional Bjorken flow and considering only massless gluonic degrees of freedom. We leave, for the future, extensions to more realistic geometries $[19,20,25]$ and inclusion of quark degrees of freedom $[42,43]$.

We thank A. Mazeliauskas for useful discussions. We also thank the Ohio Supercomputer Center under the auspices of Project No. PGS0251. M. S. and D. A. were supported by the U.S. Department of Energy, Office of Science, Office of Nuclear Physics Award No. DESC0013470.

[1] R. Averbeck, J. W. Harris, and B. Schenke, Heavy-ion physics at the LHC, in The Large Hadron Collider: Harvest of Run 1 (Springer, Cham, 2015), pp. 355-420.

[2] S. Jeon and U. Heinz, in Quark-Gluon Plasma 5, edited by X.-N. Wang (World Scientific, Singapore, 2016), pp. 131-187.

[3] P. Romatschke and U. Romatschke, Relativistic Fluid Dynamics In and Out of Equilibrium, Cambridge Monographs on Mathematical Physics (Cambridge University Press, Cambridge, England, 2019).

[4] D. Teaney, Phys. Rev. C 68, 034913 (2003).

[5] D. A. Teaney, Viscous hydrodynamics and the quark gluon plasma, in Quark-Gluon Plasma 4 (World Scientific, Singapore, 2010) pp. 207-266.

[6] K. Dusling, G. D. Moore, and D. Teaney, Phys. Rev. C 81, 034907 (2010).

[7] P. M. Chesler and L. G. Yaffe, Phys. Rev. D 82, 026006 (2010).

[8] A. Kurkela and Y. Zhu, Phys. Rev. Lett. 115, 182301 (2015).

[9] P. M. Chesler, J. High Energy Phys. 03 (2016) 146.

[10] A. Kurkela, A. Mazeliauskas, J.-F. Paquet, S. Schlichting, and D. Teaney, Phys. Rev. C 99, 034910 (2019).

[11] A. Kurkela, U. A. Wiedemann, and B. Wu, Eur. Phys. J. C 79, 759 (2019).

[12] A. Kurkela, U. A. Wiedemann, and B. Wu, Eur. Phys. J. C 79, 965 (2019).

[13] M. P. Heller and M. Spalinski, Phys. Rev. Lett. 115, 072501 (2015).

[14] L. Keegan, A. Kurkela, P. Romatschke, W. van der Schee, and Y. Zhu, J. High Energy Phys. 04 (2016) 031.

[15] M. P. Heller, A. Kurkela, M. Spaliski, and V. Svensson, Phys. Rev. D 97, 091503(R) (2018).

[16] W. Florkowski, M. P. Heller, and M. Spalinski, Rep. Prog. Phys. 81, 046001 (2018).

[17] P. Romatschke, Phys. Rev. Lett. 120, 012301 (2018).

[18] M. Spaliski, Phys. Lett. B 776, 468 (2018).

[19] P. Romatschke, J. High Energy Phys. 12 (2017) 079.
[20] A. Behtash, C. N. Cruz-Camacho, and M. Martinez, Phys. Rev. D 97, 044041 (2018).

[21] W. Florkowski, E. Maksymiuk, and R. Ryblewski, Phys. Rev. C 97, 024915 (2018).

[22] W. Florkowski, E. Maksymiuk, and R. Ryblewski, Phys. Rev. C 97, 014904 (2018).

[23] M. Strickland, J. Noronha, and G. S. Denicol, Phys. Rev. D 97, 036020 (2018).

[24] D. Almaalol and M. Strickland, Phys. Rev. C 97, 044911 (2018).

[25] G. S. Denicol and J. Noronha, Phys. Rev. D 99, 116004 (2019).

[26] A. Behtash, C. N. Cruz-Camacho, S. Kamata, and M. Martinez, Phys. Lett. B 797, 134914 (2019).

[27] M. Strickland, J. High Energy Phys. 12 (2018) 128.

[28] M. P. Heller and V. Svensson, Phys. Rev. D 98, 054016 (2018).

[29] A. Behtash, S. Kamata, M. Martinez, and H. Shi, J. High Energy Phys. 07 (2020) 226.

[30] M. Strickland and U. Tantary, J. High Energy Phys. 10 (2019) 069.

[31] S. Jaiswal, C. Chattopadhyay, A. Jaiswal, S. Pal, and U. Heinz, Phys. Rev. C 100, 034901 (2019).

[32] A. Kurkela, W. van der Schee, U. A. Wiedemann, and B. Wu, Phys. Rev. Lett. 124, 102301 (2020).

[33] C. Chattopadhyay and U. W. Heinz, Phys. Lett. B 801, 135158 (2020).

[34] J. Brewer, L. Yan, and Y. Yin, arXiv:1910.00021.

[35] M. Attems, J. Casalderrey-Solana, D. Mateos, D. Santos-Olivn, C.F. Sopuerta, M. Triana, and M. Zilho, J. High Energy Phys. 01 (2017) 026.

[36] P. B. Arnold, G. D. Moore, and L. G. Yaffe, J. High Energy Phys. 01 (2003) 030.

[37] M. C. Abraao York, A. Kurkela, E. Lu, and G. D. Moore, Phys. Rev. D 89, 074036 (2014).

[38] R. Baier, A. H. Mueller, D. Schiff, and D. T. Son, Phys. Lett. B 502, 51 (2001).

[39] A. H. Mueller, Phys. Lett. B 475, 220 (2000).

[40] A. Kurkela and Y. Zhu, Phys. Rev. Lett. 115, 182301 (2015).

[41] P. B. Arnold, G. D. Moore, and L. G. Yaffe, J. High Energy Phys. 05 (2003) 051.

[42] A. Kurkela and A. Mazeliauskas, Phys. Rev. Lett. 122, 142301 (2019).

[43] A. Kurkela and A. Mazeliauskas, Phys. Rev. D 99, 054018 (2019).

[44] P. Romatschke and M. Strickland, Phys. Rev. D 68, 036004 (2003).

[45] L. Keegan, A. Kurkela, A. Mazeliauskas, and D. Teaney, J. High Energy Phys. 08 (2016) 171.

[46] A. Mueller, Nucl. Phys. B572, 227 (2000).

[47] Y. V. Kovchegov, Nucl. Phys. A692, 557 (2001).

[48] T. Lappi, Phys. Lett. B 703, 325 (2011).

[49] See Supplemental Material at http://link.aps.org/ supplemental/10.1103/PhysRevLett.125.122302 for plots and brief discussion of a larger set of scaled moments in order to provide further evidence of the findings in the main part of the Letter, which includes Ref. [50].

[50] M. A. York and G. D. Moore, Phys. Rev. D 79, 054011 (2009). 
[51] A. Kurkela and Y. Zhu, Phys. Rev. Lett. 115, 182301 (2015).

[52] G. Giacalone, A. Mazeliauskas, and S. Schlichting, Phys. Rev. Lett. 123, 262301 (2019).

[53] M. Spaliński, Phys. Lett. B 784, 21 (2018).

[54] A. Kurkela and G. D. Moore, J. High Energy Phys. 11 (2011) 120.

[55] J. Berges, K. Boguslavski, S. Schlichting, and R. Venugopalan, Phys. Rev. D 89, 074011 (2014).

[56] J. Berges, K. Boguslavski, S. Schlichting, and R. Venugopalan, Phys. Rev. D 89, 114007 (2014).

[57] J. Berges, K. Boguslavski, S. Schlichting, and R. Venugopalan, Phys. Rev. Lett. 114, 061601 (2015).

[58] A. Mazeliauskas and J. Berges, Phys. Rev. Lett. 122, 122301 (2019).

[59] W. Florkowski, R. Ryblewski, and M. Strickland, Nucl. Phys. A916, 249 (2013).

[60] W. Florkowski, R. Ryblewski, and M. Strickland, Phys. Rev. C 88, 024903 (2013).

[61] G. Baym, Phys. Lett. 138B, 18 (1984).

[62] G. Baym, Nucl. Phys. A418, 525C (1984).
[63] G. S. Denicol, T. Koide, and D. H. Rischke, Phys. Rev. Lett. 105, 162501 (2010).

[64] G. S. Denicol, J. Noronha, H. Niemi, and D. H. Rischke, Phys. Rev. D 83, 074019 (2011).

[65] W. Florkowski and R. Ryblewski, Phys. Rev. C 83, 034907 (2011).

[66] M. Martinez and M. Strickland, Nucl. Phys. A848, 183 (2010).

[67] L. Tinti and W. Florkowski, Phys. Rev. C 89, 034907 (2014).

[68] M. Alqahtani, M. Nopoush, and M. Strickland, Prog. Part. Nucl. Phys. 101, 204 (2018).

[69] P. Bozek and W. Broniowski, Phys. Rev. C 88, 014903 (2013).

[70] C. Shen, J.-F. Paquet, G. S. Denicol, S. Jeon, and C. Gale, Phys. Rev. C 95, 014906 (2017).

[71] M. Alqahtani, M. Nopoush, and M. Strickland, Phys. Rev. C 95, 034906 (2017).

[72] R. D. Weller and P. Romatschke, Phys. Lett. B 774, 351 (2017).

[73] H. Mntysaari, B. Schenke, C. Shen, and P. Tribedy, Phys. Lett. B 772, 681 (2017).

[74] M. Strickland, Nucl. Phys. A982, 92 (2019). 